McGill Law Journal

Revue de droit de McGill

\title{
Une justice fraternelle : éléments de la pensée de Charles Doherty Gonthier
}

\section{Fabien Gélinas}

Volume 55, numéro 2, july 2010

URI : https://id.erudit.org/iderudit/045091ar

DOI : https://doi.org/10.7202/045091ar

Aller au sommaire du numéro

Éditeur(s)

McGill Law Journal / Revue de droit de McGill

ISSN

0024-9041 (imprimé)

1920-6356 (numérique)

Découvrir la revue

Citer cet article

Gélinas, F. (2010). Une justice fraternelle : éléments de la pensée de Charles Doherty Gonthier. McGill Law Journal / Revue de droit de McGill, 55(2), 357-363.

https://doi.org/10.7202/045091ar d'utilisation que vous pouvez consulter en ligne. 
McGill Law Journal Revue de droit de McGill

\title{
UNE JUSTICE FRATERNELLE : ÉLÉMENTS DE LA PENSÉE DE CHARLES DOHERTY GONTHIER
}

\author{
Fabien Gélinas*
}

\section{Introduction}

On a rendu un hommage vibrant au gentilhomme humaniste qu'était Charles Gonthier en relevant tout particulièrement son sens profond de la justice, sa courtoisie exemplaire en toutes circonstances, son sens quasi légendaire de la mesure, ses prises de position nuancées sur les questions difficiles, sa manière d'incarner le bijuridisme, sa défense des plus hauts standards d'intégrité pour la magistrature et son souci de protéger les personnes vulnérables tout en insistant sur les devoirs et la responsabilité des individus ${ }^{1}$. Il y aurait beaucoup à dire sur la vie et la pensée de Charles Gonthier, que j'ai eu le privilège de connaître pendant près de vingt ans. Ce texte est destiné à lui rendre hommage en retraçant les linéaments de sa réflexion sur les rapports entre le droit, la moralité et la communauté.

\section{Droit et moralité}

Charles Gonthier a toujours été reconnu comme un homme profondément moral. Sa réserve naturelle ainsi qu'un sens inné du respect de l'autre l'ont toutefois toujours préservé de la tentation de jouer les moralisateurs $^{2}$. Ses années passées à la Cour suprême du Canada et les réflexions qu'elles ont pu susciter sur les rapports entre le droit (et le rôle du juge) et la moralité sont venues selon moi renforcer dans son esprit les raisons d'une position au départ adoptée par tempérament.

* Professeur agrégé à la Faculté de droit et à l'Institut de droit comparé de l'Université McGill. Le professeur Gélinas a agi comme auxiliaire juridique auprès de l'honorable Charles Doherty Gonthier de 1989 à 1990.

$$
\text { (C) Fabien Gélinas } 2010
$$

Citation: (2010) 55 McGill L.J. 357 Référence : (2010) 55 R.D. McGill 357

1 Voir notamment Michel Robert, « Hommage à l'honorable juge Charles Gonthier à sa retraite de la Cour supême du Canada » (2003) 48 R.D. McGill 175 à la p. 177.

$2 \quad$ Ibid. 
Des jugements de Charles Gonthier, ainsi que de ses écrits parus avant et après son départ de la Cour, on peut tirer deux propositions fondamentales sur les rapports entre le droit et la moralité.

\section{A. Le droit ne peut imposer une conception, soit-elle majoritaire, de la moralité}

Fortement attiré par certains éléments des théories politiques dites " communautaristes", Charles Gonthier est néanmoins toujours resté attaché aux fondements des théories politiques associées au libéralisme.

Réfléchissant sur ces questions à la toute fin de sa carrière de juge, Charles Gonthier affirmait d'ailleurs considérer les divisions entre le communautarisme et le libéralisme comme artificielles ${ }^{3}$. Selon lui, les deux perspectives théoriques partagent un seul et même objectif ultime, celui d'assurer la dignité de l'individu au sein de sa communautét. Le reste est une question de degré. C'est ainsi qu'un document comme la Charte canadienne des droits et libertés ${ }^{5}$ peut être considéré comme le reflet à la fois de principes libéraux et de principes communautaristes ${ }^{6}$.

À la base, et conformément aux principes du libéralisme, le droit ne saurait imposer la moralité telle qu'elle est conçue par la majorité des citoyens : "The law cannot impose the majority's moral views of the "good life" on the rest of the population ${ }^{7}$. Le droit ne pourrait d'ailleurs se maintenir dans un contexte de diversité culturelle considérable en prenant systématiquement position sur toutes les questions de moralité.

Mais le droit protège tout de même la communauté dont il émane des menaces que présentent certains comportements et activités. Le droit revendique en effet le contrôle des comportements et activités qu'il ne peut tolérer en raison de la menace fondamentale qu'ils présentent pour la communauté et son environnement moral. Pour autant que le fonctionnement de l'ordre juridique se trouve menacé, cette position demeure dans la lignée des préceptes libéraux, qui admettent que le droit doit endosser certains éléments de la moralité pour maintenir son effectivité. Mais il y a plus.

3 Voir notamment Charles D. Gonthier, «Law and Morality» (2003) 29 Queen’s L.J. 408 à la p. 412 [Gonthier, « Law»].

4 Ibid.

5 Partie I de la Loi constitutionnelle de 1982, constituant l'annexe B de la Loi de 1982 sur le Canada (R.-U.), 1982, c. 11 [Charte].

6 Voir Gonthier, « Law », supra note 3 à la p. 416.

$7 \quad$ Ibid. à la p. 414. 
En effet, dans les limites imposées par "une société pluraliste» où "les divers segments de la population ont maintes conceptions différentes de ce qui est bien", il est toujours légitime pour l'État d'intervenir pour protéger "les conceptions fondamentales de la moralité »8. Ces dernières se démarquent de la moralité au sens large en ce qu'elles se rattachent à des préoccupations sociales concrètes (et non seulement à une aversion non fondée pour une opinion ou un comportement) et font l'objet d'un consensus au sein de la population, exigence qui isole le droit constitutionnel des positions prises par une simple majorité de la population ${ }^{9}$.

\section{B. Le droit doit être moral pour être efficace mais n'est pas garant de l'amélioration du tissu moral de la société}

Cette seconde proposition se veut d'abord essentiellement descriptive de ce que le droit ne saurait en pratique assurer sa fonction régulatrice sans refléter un contenu moral minimum. Charles Gonthier reprend ce constat du philosophe H.L.A. Hart, plus précisément d'un passage tiré de l'ouvrage The Concept of Law :

In the absence of this [moral] content men, as they are, would have no reason for obeying voluntarily any rules; and without a minimum of co-operation given voluntarily by those who find that it is in their interest to submit to and maintain the rules, coercion of others who would not voluntarily conform would be impossible ${ }^{10}$.

Il suffira ici de faire mention des exemples de la bonne foi et des obligations fiduciaires en droit privé pour montrer l'intégration par le droit d'éléments de la moralité bien au-delà des matières criminelles, où le phénomène est encore plus évident. Charles Gonthier a toujours été favorable à cette intégration en tant que manifestation d'une conception téléologique du droit palliant aux excès du formalisme juridique.

Le constat de Hart, bien que largement partagé, est moins anodin qu'il n'y paraît, car il associe implicitement mais de manière significative la moralité aux conventions sociales telles qu'observées dans la réalité. C'est à cette notion conventionnelle de la moralité que nous ramène l'idée de consensus évoquée plus haut.

La moralité conventionnelle s'inscrit dans un rapport dialogique obligé avec le droit en évolution, rapport dont une illustration frappante nous est donnée dans la célèbre affaire Rodriguez c. Colombie-Britannique (Procu-

8 R. c. Butler, [1992] 1 R.C.S. 452 aux pp. 523-24, 89 D.L.R. (4e) 449, juge Gonthier, opinion concurrente.

$9 \quad$ Ibid.

10 H.L.A. Hart, The Concept of Law, Oxford, Clarendon Press, 1994 à la p. 189, tel que cité dans Gonthier, "Law », supra note 3 à la p. 414. 
reur général) ${ }^{11}$, où une victime de la maladie de Lou Gehrig a tenté sans succès de faire reconnaître par la Cour suprême du Canada un droit constitutionnel au suicide assisté. L'intérêt de cette décision, à laquelle le juge Gonthier a participé, repose principalement sur le critère du consensus social, qui semble avoir été déterminant pour la Cour dans son articulation du droit constitutionnel. En tenant compte des valeurs véhiculées par la Charte telles la vie, la liberté et la sécurité de la personne humaine, la Cour a en effet estimé qu'aucun consensus social sur la reconnaissance d'un droit au suicide assisté n'avait encore pris forme.

Mais la moralité conventionnelle qui nourrit ainsi ouvertement la norme juridique ne représente qu'un aspect limité de l'univers de la moralité. La moralité conventionnelle que le droit se permet d'intégrer n'est après tout que le dénominateur commun d'une quantité de conceptions et de systèmes moraux présents dans la société. Cette constatation explique bien pourquoi et à quel point le potentiel du droit comme vecteur d'amélioration de l'environnement moral de la société est limité.

Le droit se cantonne donc dans une position de réserve et d'humilité face aux conceptions morales et c'est ce qui lui assure une légitimité suffisante pour imposer le respect des standards modestes et conventionnels dont dépend son effectivité. Dans l'articulation de ces standards, Charles Gonthier s'est toujours inquiété du rôle, prépondérant en apparence, de la liberté et de l'égalité conçues comme droits individuels.

\section{Droit et communauté}

Le second thème que je me propose d'aborder brièvement ici concerne les rapports entre le droit et la communauté dont il émane. Pour Charles Gonthier, l'articulation du discours politico-juridique dominant autour des droits à la liberté et à l'égalité a toujours posé problème dans la représentation de nos valeurs et de nos institutions. Cette préoccupation s'énonce aisément en rappelant le texte de l'article premier de la Déclaration universelle des droits de l'Homme ${ }^{12}$ qu'il aimait citer:

Tous les êtres humains naissent libres et égaux en dignité et en droits. Ils sont doués de raison et de conscience et doivent agir les uns envers les autres dans un esprit de fraternité [nos italiques] ${ }^{13}$.

11 [1993] 3 R.C.S. 519, 107 D.L.R. (4e) 342.

12 Voir par ex. Charles D. Gonthier, «Liberty, Equality, Fraternity: The Forgotten Leg of the Trilogy, or Fraternity: The Unspoken Third Pillar of Democracy" (2000) 45 R.D. McGill 567 à la p. 569 [Gonthier, « Liberty»].

13 Déclaration universelle des droits de l'Homme, Rés. AG 217(III), Doc. off. AG NU, 3e sess., supp. no13, Doc. NU A/810 (1948) 71, art. 1. 
Les mots " doivent " et " fraternité " demeuraient pour lui trop largement méconnus et ce, au grand péril de l'évolution à long terme de la justice dans nos sociétés. Il y a là d'abord l'idée du devoir comme face cachée des droits : ces derniers ne sont qu'une vaste chimère s'il n'y a point de devoirs qui y correspondent. Il y a en second lieu l'idée qu'une proclamation de la liberté et de l'égalité des êtres humains prend son sens, au premier chef, au sein d'une communauté.

\section{A. Sans devoirs point de droits}

Selon Charles Gonthier, " [l]e droit de chacun n'est jamais dissocié de l'obligation de chacun envers les autres ; l'acte posé est toujours relié à la responsabilité qu'il engendre; l'erreur est toujours appréciée dans son contexte et dans ses causes ${ }^{\prime 14}$. C'est cette perspective d'ensemble qui explique l'enthousiasme relatif avec lequel Charles Gonthier appréhendait la "Révolution des droits" que l'on a pu associer à l'entrée en vigueur de la Charte au Canada ${ }^{15}$.

Pour lui, « [s]ouvent, l'action des tribunaux en vertu de la Charte a eu pour effet de concrétiser certaines valeurs fondamentales qui soustendaient auparavant de manière implicite le droit constitutionnel canadien ${ }^{16}$. Aussi, bien qu'il ait été soucieux d'aménager une marge de manœuvre suffisante au législateur dans la poursuite des politiques publiques, il était tout à fait disposé à déclarer les lois inconstitutionnelles lorsque les restrictions aux droits garantis le justifiaient ${ }^{17}$. Sa préoccupation avait trait non pas au principe de la garantie constitutionnelle des droits de l'individu, mais bien à l'effet potentiel de cette garantie sur le sens et la conscience du devoir et des responsabilités. Selon lui, la Charte devrait " donner réalité à la participation éclairée du public aux débats fondamentaux qui orientent la société $»^{18}$. Il devrait en résulter un développement du sens du devoir et des responsabilités chez les justiciables.

L’enjeu, ici, est celui du degré de coercition nécessaire au fonctionnement d'une société protectrice de libertés. Sans prise de conscience de ce qui est dû aux autres et à la communauté, il ne reste que la force pour assurer la cohésion et le maintien d'une communauté de droit.

Robert, supra note 1 à la p. 177.

15 Voir Alain-Robert Nadeau, «Les libertés fondamentales et l'art du droit» (2003) $35 \mathrm{~J}$. Barreau 14 à la p. 14.

16 Ibid.

17 Voir Michel Morin, «Charles D. Gonthier, un juriste épris de justice» Le Devoir [de Montréal] (21 juillet 2009) A6.

18 Nadeau, supra note 15 à la p. 14. 
La justesse de cette préoccupation n'a jamais été aussi évidente qu'elle ne l'est aujourd'hui, à l'heure où les juridictions occidentales œuvrent à l'articulation ou au renforcement de garanties juridiques relatives aux droits et libertés fondamentales dans la sphère privée, où le débiteur de l'obligation n'est plus l'État mais bien l'individu. Charles Gonthier rappelait d'ailleurs avec justesse que la Charte des droits et libertés de la personne $^{19}$ du Québec, un précurseur à cet égard, avait dûment et naturellement pris acte de l'importance du devoir envers les autres en imposant à tous une obligation expresse d'assistance aux personnes en danger ${ }^{20}$. Il s'agissait là, pour lui, de l'une des nombreuses manifestations du principe de fraternité dans l'ordre juridique canadien.

\section{B. Sans fraternité point d'égale liberté}

$\mathrm{Au}$ crépuscule de sa brillante carrière de juge, Charles Gonthier a consacré une énergie considérable au développement et à l'exposition de la notion de fraternité, qu'il jugeait essentielle au bon fonctionnement d'une démocratie ${ }^{21}$. La fraternité évoque des valeurs telles que l'empathie, la coopération, l'engagement, la responsabilité, la confiance, le fair play et l'équité22.

Parmi les nombreux rôles importants joués par le principe de fraternité, on trouve celui de passerelle qui nous lie à la fois au passé et aux générations qui vont nous succéder. On peut soupçonner que cette idée est à la source du choix fait par Charles Gonthier de consacrer ses énergies, après son départ de la Cour suprême du Canada, au développement durable. Pour lui, "Forging a relationship between generations in a community is not rooted in liberty or equality, but rather, fraternity $»^{23}$.

La fraternité est toutefois bien ancrée dans le présent. Valeur exprimée dans la Déclaration universelle des droits de l'Homme comme pendant de la liberté et de l'égalité, et pourtant largement oubliée dans le discours des droits de l'homme, la fraternité avait pour Charles Gonthier le statut de principe sous-jacent de l'ordre juridique et constitutionnel canadien.

Comme il a réussi à le démontrer, on peut en effet envisager ce principe comme donnant lieu à une foule de règles et de théories tant en droit public qu'en droit privé, et tant en droit civil qu'en common law ${ }^{24}$. La re-

L.R.Q. c. C-12, art. 2.

Voir Gonthier, «Liberty », supra note 12 à la p. 579.

Ibid. à la p. 589.

Ibid. à la p. 572.

Ibid. à la p. 573 .

Ibid. 
connaissance de ces règles et théories par le droit positif donne en retour un poids considérable à la fraternité comme principe juridique structurant, un principe qui doit prendre sa juste place aux côtés de la liberté et de l'égalité si l'on ose concevoir ces concepts dans la durée.

Pour Charles Gonthier, la fraternité est le ciment qui rattache la liberté et l'égalité à une société civile ${ }^{25}$. Acceptant cette idée, la question de l'identification ou de la définition de cette société civile, ou communauté, demeure toutefois ouverte. La fraternité se développe d'abord au sein de la famille, puis s'articule autour des rapports humains et se formalise dans les institutions. À l'heure où l'on s'interroge sur le rôle de l'État et de son droit dans la régulation sociale mondialisée, les questions soulevées par Charles Gonthier sur le lieu de déploiement du principe de fraternité sont, encore ici, plus que jamais d'actualité.

La position de réserve et d'humilité adoptée par l'ordre juridique canadien face aux conceptions morales lui semblait à cet égard tout à fait pertinente dans le développement du "village global ", qui passerait par l'émergence de standards universels de tolérance, un phénomène bien sûr amorcé par la Déclaration universelle des droits de l'Homme, et qui semble s'accélérer aujourd'hui.

\section{Conclusion}

Sans être orientalisant, Charles Gonthier, l'homme comme le juge incomparable qu'il fut, semblait bien avoir découvert les trois perles de la sagesse taoïste : la compassion, la modération et l'humilité. Il nous laisse un héritage hors du commun qui nous engage tous à remettre en rapport dialogique nos théories des droits institutionnalisés et le devoir de chacun de rendre justice à son prochain et à sa communauté.

$25 \quad$ Ibid. à la p. 569. 